\title{
The Deployment of Resource Pool Based on Hyper-V Virtualization in Cloud Computing
}

\author{
Shuqian Liu, Pengdong Gao, Kaihui Mu, Yongquan Lu \\ High Performance Computing Center, Communication University of China, Beijing 100024, China \\ liushuqian2007@126.com,pd_gao@cuc.edu.cn,yqlu@cuc.edu.cn
}

\begin{abstract}
To build a high-efficient cloud platform for virtual resources, we deployed a virtual resource pool based on Hyper-V Virtualization to meet people's requirements. In this way, users can get a free VM from the pool in less time compared with creating a new one. A VM usually includes an operating system, the network to communicate with cloud platform and end-users, the shared storage space and other configurations. It will be presented that the deployment process of the resource pool and the infrastructure of cloud platform in this paper. The time to wait for an available VM will take just about $1 / 3$ of the original time after using resource pool.

Index Terms - Resource pool, hyper-v, virtualization, cloud computing, cloud storage
\end{abstract}

\section{Introduction}

Combining virtualization technology with cloud computing has been a fashion nowadays. Cloud is a huge container that can merge different virtualization technologies and applications together through the Internet. Considering the high consumption in the management of physical PCs or servers, increasingly more individuals and enterprises turn their attention to cloud services that can be offered through the internet. End-users tend to use virtual resources for large-scale data processing, computing, animation rendering and data storage. And these services must depend on higher network speed, more convenient interaction design and the high availability of services. Providing consistent security services in on-demand provisioned Cloud infrastructure services is of primary importance due to the multi-tenant and potentially multi-provider nature of Cloud Infrastructure [1].

Recent years, cloud computing is building a larger market share depending on its advantages of rapid deployment, ease of maintenance, and relatively low cost. Cloud computing: A large-scale distributed computing paradigm that is driven by economies of scale, in which a pool of abstracted, virtualized, dynamically-scalable, managed computing power, storage, platforms, and services are delivered on demand to external customers over the Internet[2]. We can see that cloud computing is closely related with virtualization, storage and network from this definition. On one hand, as the core technology of cloud computing, virtualization makes it to manage the pool of potential computing resources. Cloud resources are usually isolated on the Internet, such as servers, storage, applications and other services. On the other hand, the network plays a role of a bridge to build a connection between cloud providers and customers. From a service provisioning perspective, Cloud services consist of not only computing functions provided by
Cloud infrastructure but also communications functions offered by the Internet [3].

Therefore, the combination of cloud infrastructure platform and virtualization is a key point when providing customers services. When it comes to the Virtualization technology, three main manufacturers automatically cross our mind, the Microsoft, VMware and Citrix. Obviously, there are two major differences among them. The first one comes to the different Remote Access Protocol, including the RDP (Remote Desktop Protocol) for Microsoft, the ICA (Independent Computing Architecture) for Citrix and the PCoIP protocol for VMware. The second difference comes to the virtualization technology they use for servers. Microsoft uses the Hyper-V virtualization, VMware uses its own vSphere and Citrix uses a mix of XenServer, Hyper-v and vSphere.

Although prices of electronic products have been reduced a lot, it's still a great expenditure for individuals and enterprises while chasing the high-level configuration of PCs. Consequently, a virtualization solution providing high-available VMs is extremely necessary. In this paper, we will mainly present the deploying process of the Virtual Machine Pool based on the Hyper-v virtualization with its Plug-in in the cloud platform. Hyper-v is published as a component on Windows Server 2008 and Windows Server 2012 operating system. Besides, it provides a basic virtualization platform and makes it easy to migrate to Cloud-client.

There are four essential elements in an available Hyper-V VM, as shown in figure 1:

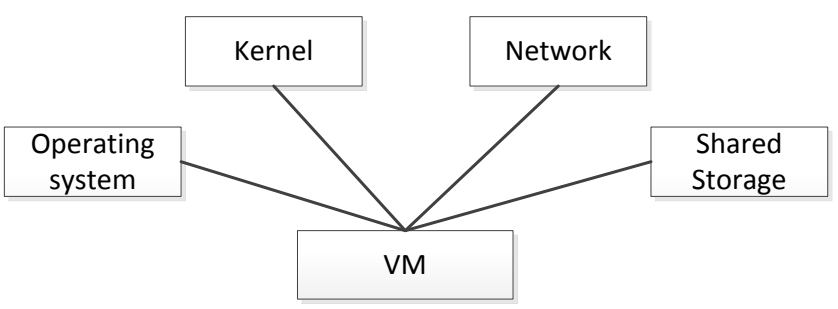

Figure 1 The infrastructure of cloud platform

A VM owns its operating system, and it can be Windows, Linux or other system. A kernel is a micro core for management. The network mainly means the IP address, MAC address for net connecting. For a cloud user, he may have a large data, so we managed to offer an access to the user's shared storage on the cloud platform. 


\section{The Architecture of the Cloud Service and Virtualization}

In order to offer customers an efficient and reliable experience when they require a VM from on the internet, we need the support of hardware facilities to offer the essential infrastructure, a high-speed and safe network environment to build the connection between customers and cloud services, an available resource pool to provide actual virtual resources, besides, an available access to the cloud services is necessary.

The fundamental architecture of our cloud platform is designed as figure 2:

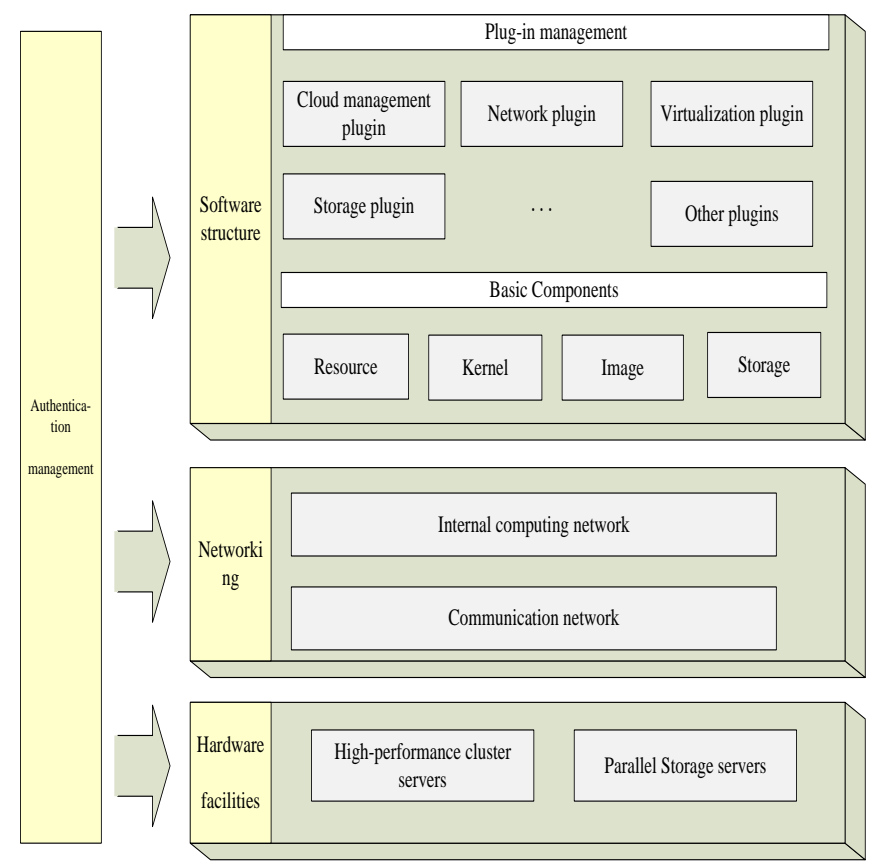

Figure 2 The infrastructure of cloud platform

\section{1) Hardware facilities}

Cloud computing is welcome nowadays for its high stability and efficiency, depending on the support of the powerful facilities to a large extent. High-performance cluster servers are an essential part in a cloud platform. It makes it to manage more storage nodes and computing nodes in a parallel way and provide customers a high-efficiency and dependable service. At the same time, the processing performance of servers can be improved effectively while a massive amount of data communication is produced. There exists an interchangeable space between Cluster system and virtualization system to a certain extent. Virtualization technology can improve the hardware utilization by virtualizing a single server into multiple virtual computing resources, while clustering technology centralizes multi-server performance together to form a "super computer" to provide high-performance services. Both technologies manage to utilize the system resources more reasonable and fully by optimizing and re-mix to the load of servers.

Parallel storage servers in cloud computing provide a high-availability storage environment, improving the throughput and speed when transfer users' data.

\section{2) Networking}

A high-speed and secure network environment is always the most important factor for customers to consider when applying cloud services. Here in our architecture, there exist two kinds of network. The first one is the internal computing network which is used for the control and data transaction between servers. Due to the demand of high-speed transaction for mass data, the private network is always required, such as InfiniBand. InfiniBand is a switched fabric computer network communications link used in high-performance computing and enterprise data centers [4].

As to the communication network, it is mainly used for the communication between end-users and cloud.

Software structure

With the development of software engineering and object-oriented methodology based software design, plug-in technology is burgeoning along with certain features of object-oriented methodology [5]. As a result of frequent demand changes of customers and more and more extending functions, the software framework with plug-in technology applied turns out to be a perfect solution in software deployment. We apply the plug-in technology in our cloud platform for networks, storage, high availability, virtualization, cloud services, and other products.

The cloud infrastructure platform has a pluggable architecture which is designed to provide comprehensive support for continuous integration. The main function of the basic framework is to manage different plug-ins, which makes it possible to allow several plug-ins running in parallel without interfering with each other. The structure is separated into two parts. The basic components mainly include resource, kernel, image and storage, which are the essential elements in the cloud platform. A resource includes all kinds of CPUs and memories of physical system, host machines or virtual machines, communications are carried out with certain resources according to different interfaces. A kernel here means the Linux kernel assigned to a resource, a SYSLINUX derivative, for booting Linux from a network server using a network ROM conforming to the Intel PXE (Pre-Execution Environment) specification [6]. The image in this structure means the root-filesystem of operating system, stored in the parallel storage servers independently. The storage component consists of computing resources integrated by a network connection, providing the exact storage location of the system image.

The Plug-in management is the body of cloud infrastructure platform. As plug-in has larger granularity of software reuse that has higher level of encapsulation method [5], it becomes easy to enhance, change and extend functions without modifying the main part of software [5]. And thanks to this characteristic, various virtualization technologies are easy to be integrated to the platform. Therefore, we integrated the cloud management, network, virtualization, storage and other services together in the same platform as independent plug-ins. Plugin-ins in cloud platform make it possible to deploy resource pool based on Hyper-V virtualization. 


\section{3) Authentication mechanism}

Only if they are validated through the authentication system, which provides a security mechanism to avoid illegal users gaining access cloud services, and thus ensure the data and network security.

\section{The Deployment Process of Resource Pool}

A Virtual Machine is the same as a physical PC. It owns almost every component a physical PC has, except the virtual characteristics. Generally, we have to create an idle virtual computer first, and then install the operating system for it, and importantly, we must manage to build the connection between the VMs and users, thus allow people to gain access to login the VM. However, this process usually takes more than 6 minutes as we tested, which is a little longer for users to wait. So, we try to deploy the resource pool to reduce the time interval to a minimum that users can bear.

Several kinds of virtualization techniques are usual used nowadays, such as VMware, Virtual Box, Virtual PC, and now the Hyper-V technique. Well in our platform, we put emphasis on the virtualization of Hyper-V. Hyper-V Server is a dedicated stand-alone product that contains the hypervisor, Windows Server driver model, virtualization capabilities, and supporting components such as failover clustering, but does not contain the robust set of features and roles as the Windows Server operating system [7]. The hyper-v virtualization is characterized by its easier deployment, and what we need to do is to automatize the deployment.

\section{1) Deployment of the resource pool}

The resource pool means to provide cloud users a series of virtual machines (VM) with various configurations. Every registered user can apply their VMs according to their requirements. The deployment framework is shown as figure3:

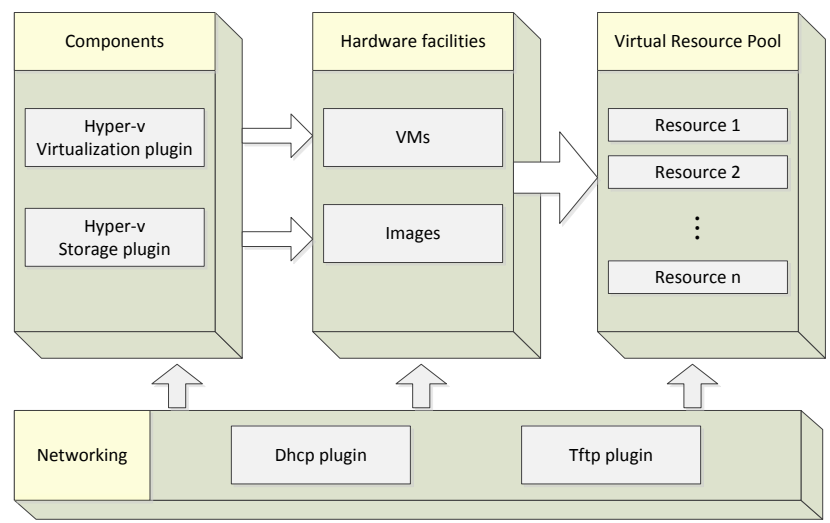

Figure 3 The deployment framework of resource pool

Firstly, the Hyper-V virtualization plugin is used to call its interface to produce virtual machines. The cloud platform has built a connection between the plugin management system and host servers. All the Windows server hosts are deployed with Hyper-v component, thus the Hype-v virtualization plugin is able to send commands to the Hyper-v manager on the server to create and manage a new VM. The commands are sent to servers and then call the powershell and python scripts to execute operating commands. The command will include the CPU number, memory size requested by users, in addition, the GPU acceleration will be offered to users when they use the VM.

Secondly, the Hyper-v storage plugin will call certain interface to send clone command to produce the image file on storage server. Actually, all image files and user data are stored in a parallel storage cluster which has a connection with the Hyper-v host servers through the InfiniBand network.

Then the image will be attached to the VM, thus constituting a resource, and after the VM start from image file, the script in it will send a signal to cloud platform to declare as a new and free VM. Then, a new resource in pool is produced. Cloud users can get access to the cloud portal to apply resources eventually.

Cloud platform will produce several kinds of resources to users which will be put into the "resource pool", waiting for being requested.

\section{2) User-application for resource}

Every new resource in pool will be available to registered cloud users. They can choose to apply new resource or re-use their used resources, while the system can attach any available image to a free VM as the images and VMs are managed separately. With the on-line effective cloud storage system, users needn't to worry about obliterating data. Only in a few minutes, a virtual resource is shown to the end-user, the process is shown as figure 4 :

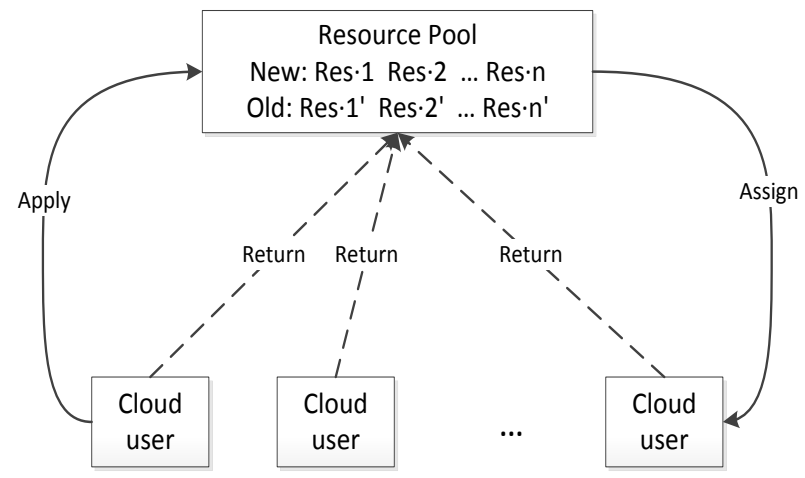

Figure 4 The application process for users

Step 1: Applying. A new resource means a free VM that has not been applied since it was put in pool. A used resource refers to a VM which has been used by a certain user, after it was de-provisioned, the user can apply it again without data lose.

Step 2: Resource distribution. A free resource (VM) that matched user's request info will be assigned to him.

Step 3: System preparation. The cloud platform will embed user info to his requested VM through the plugins.

Step 4: Available. After the system is prepared, users can get the "ready" status from the cloud portal, and gain access to login this VM. Meanwhile, they can also gain the access to their own storage space on the VM, checking data, downloading and uploading files. 
Step 5: Return. If the resource was deleted by user, the VM will be put back to the pool, while the image is kept for user to re-use.

Actually, Step1 will be conducted many times to produce enough virtual machines. Every time we create a VM, system will choose a host server which has the lowest load. The total number of the VMs in this resource pool depends on the whole load of our cluster servers.

\section{3) Expected result}

Since the deployment of a resource pool has finished most of the work to prepare a VM, so we just need to assign an existing resource to a certain user instead of creating a new one. This should reduce the duration from user's request to available login a lot.

Here is the compare result we recorded (as shown in figure 5):

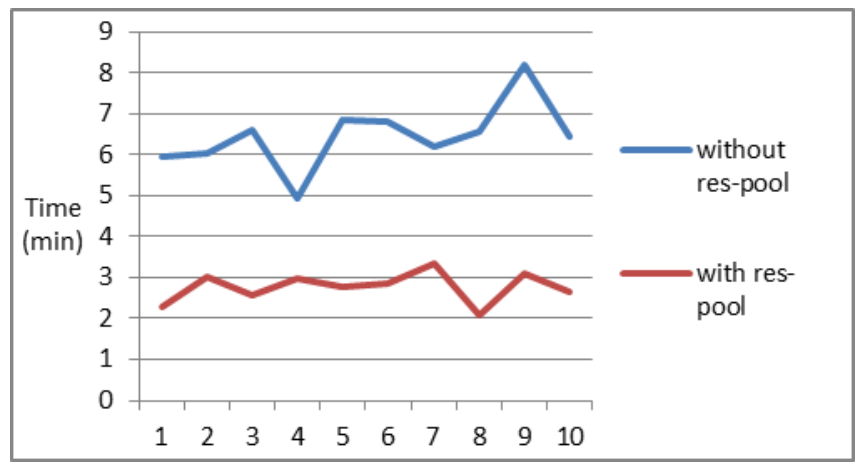

Figure 5 The compare result (the time means the total time to prepare a VM after the user's request)

From the result we can see that the time has been reduced to almost $1 / 3$ of the former one. Resource pool has helped to achieve our expectation.

\section{Conclusion}

We have introduced the architecture of the cloud platform and the plug-in management for different services in this paper. Based on the Hyper-v virtualization, we expounded the deployment process for a virtual Hyper-v resource pool and the applying process for end-users. As the result shows, the average time to offer an available VM to a cloud user has reduced to a large extent.

\section{Acknowledgment}

The authors acknowledge the financial supports by the National Key Technology Support Program (2012BAH17B03) and the Program Project of CUC (XNG1138, YXJS2012319 and YXJS2012206).

\section{References}

[1] Demchenko, Y. Canh Ngo, de Laat, C., Wlodarczyk, T.W. , Chunming Rong, and Ziegler, W., "Security Infrastructure for On-demand Provisioned Cloud Infrastructure Services" in 2011 Third IEEE International Conference on Coud Computing Technology and Science. 255-263

[2] Ian Foster, Yong Zhao, Ioan Raicu and Shiyong Lu, "Cloud computing and grid computing 360-degress compared" in Proc. 2008 Grid Computing Environment Workshop, pp. 1-10.

[3] Qiang Duan, Yuhong Yan, and Athanasios V. Vasilakos, "A Survey on Service-Oriented Network Virtualization Toward Convergence of Networking and Cloud Computing" in IEEE TRANSACTIONS ON NETWORK AND SERVICE MANAGEMENT, VOL. 9, NO. 4, DECEMBER 2012. 373-392

[4] http://en.wikipedia.org/wiki/InfiniBand

[5] Gao Lei, Fang Yu, Pan Shulin, "Software Framework Construction Based on Plug-in Technology" in 2011 International Conference on Computational and Information Sciences, 762-764

[6] http://www.syslinux.org/wiki/index.php/PXELINUX

[7] http://www.microsoft.com/en-us/server-cloud/hyper-v-server/default.as $\mathrm{px}$ 